\title{
How to detect and reduce potential sources of biases in studies of SARS-CoV-2 and COVID-19
}

\author{
Emma K. Accorsi ${ }^{1}$ (D) Xueting Qiu ${ }^{1} \cdot$ Eva Rumpler $^{1} \cdot$ Lee Kennedy-Shaffer $^{1,2} \cdot$ Rebecca Kahn $^{1} \cdot$ Keya Joshi $^{1}$. \\ Edward Goldstein ${ }^{1} \cdot$ Mats J. Stensrud ${ }^{3} \cdot$ Rene Niehus $^{1} \cdot$ Muge Cevik $^{4,5,6} \cdot$ Marc Lipsitch $^{1,7}$
}

Received: 12 November 2020 / Accepted: 4 February 2021 / Published online: 25 February 2021

(c) The Author(s) 2021

\begin{abstract}
In response to the coronavirus disease (COVID-19) pandemic, public health scientists have produced a large and rapidly expanding body of literature that aims to answer critical questions, such as the proportion of the population in a geographic area that has been infected; the transmissibility of the virus and factors associated with high infectiousness or susceptibility to infection; which groups are the most at risk of infection, morbidity and mortality; and the degree to which antibodies confer protection to re-infection. Observational studies are subject to a number of different biases, including confounding, selection bias, and measurement error, that may threaten their validity or influence the interpretation of their results. To assist in the critical evaluation of a vast body of literature and contribute to future study design, we outline and propose solutions to biases that can occur across different categories of observational studies of COVID-19. We consider potential biases that could occur in five categories of studies: (1) cross-sectional seroprevalence, (2) longitudinal seroprotection, (3) risk factor studies to inform interventions, (4) studies to estimate the secondary attack rate, and (5) studies that use secondary attack rates to make inferences about infectiousness and susceptibility.
\end{abstract}

Keywords Epidemiological biases $\cdot$ Selection bias $\cdot$ Misclassification $\cdot$ Measurement error $\cdot$ COVID-19 $\cdot$ Observational data

Emma K. Accorsi

eaccorsi@g.harvard.edu

Xueting Qiu

xuetingqiu@ hsph.harvard.edu

Eva Rumpler

erumpler@hsph.harvard.edu

Lee Kennedy-Shaffer

lkennedyshaffer@vassar.edu

Rebecca Kahn

rek160@mail.harvard.edu

Keya Joshi

keyajoshi@g.harvard.edu

Edward Goldstein

egoldste@hsph.harvard.edu

Mats J. Stensrud

mats.stensrud@epfl.ch

Rene Niehus

rniehus@hsph.harvard.edu

Muge Cevik

mc349@st-andrews.ac.uk
Marc Lipsitch

mlipsitc@hsph.harvard.edu

1 Center for Communicable Disease Dynamics, Department of Epidemiology, Harvard T.H. Chan School of Public Health, Boston, MA 02115, USA

2 Department of Mathematics and Statistics, Vassar College, Poughkeepsie, NY 12604, USA

3 Department of Mathematics, École Polytechnique Fédérale de Lausanne, Lausanne, Switzerland

4 Division of Infection and Global Health Research, School of Medicine, University of St Andrews, St Andrews, UK

5 Specialist Virology Laboratory, Royal Infirmary of Edinburgh, Edinburgh, UK

6 Regional Infectious Diseases Unit, Western General Hospital, Edinburgh, UK

7 Department of Immunology and Infectious Diseases, Harvard T.H. Chan School of Public Health, Boston, MA 02115, USA 


\section{Introduction}

Since the onset of the coronavirus disease (COVID-19) pandemic, public health scientists have worked tirelessly to provide the knowledge needed to address this new, global crisis. The pandemic has spurred an exceptional number and breadth of scientific studies [1,2], including epidemiologic ones, with the pace making it both important and challenging for researchers to design and analyze studies in the most robust way possible, and for reviewers and users to accurately evaluate the strength of evidence such studies provide. Using relevant examples from the literature, we discuss potential epidemiological biases arising in various phases of observational studies of COVID-19 and outline possible solutions.

We consider biases arising across five classes of research questions: (1) estimates of seroprevalence [3], (2) estimates of seroprotection $[4,5]$, (3) studies of risk factors for becoming infected [6], (4) estimates of the secondary attack rate [7], and (5) comparisons of secondary attack rates to make inferences about susceptibility and infectiousness [7].

\section{Seroprevalence measurement to estimate cumulative incidence}

Serological surveillance studies detect SARS-CoV-2 specific antibodies in the population and can provide an estimate of the seroprevalence-the proportion of various groups (e.g., age groups) harboring such antibodies at a single time point or, if repeated, over time. If antibodies are a marker of protection, seroprevalence may provide a direct estimate of the fraction of individuals immune to the virus, although, as we note in the next section, the protective role of antibodies against future infection remains uncertain and may wane over time [8]. If antibodies are a reliable measure of prior infection, then seroprevalence can also be used as a proxy for the cumulative incidence of infection until that time point (more precisely, until an earlier time point, because antibodies take time to rise to detectable levels after infection $[8,9])$.

As measures of cumulative incidence, seroprevalence studies can be more accurate than direct counting of case reports, especially for an infection that is often not detected due to limited testing and/or lack of symptoms and where case ascertainment rates have varied drastically over time. Yet to achieve accurate estimates from seroprevalence studies it is important to recruit a representative sample of the population of interest, and to consider measurement error, which can still create bias in a perfectly collected sample. Bias is always in reference to the specific variable which is being estimated in a statistical analysis (i.e., the estimand) so we will suppose we want to estimate the cumulative incidence of infection using seroprevalence among all people living in a particular city. We note the following considerations regarding potential biases in studies where seroprevalence is used to estimate cumulative incidence and use the terms interchangeably below.

Seroprevalence estimates may be unrepresentative of the target population when the individuals enrolled in the study are not representative of that population. The direction and magnitude of the resulting bias depend on the population for which inference of seroprevalence is being attempted, for example, all residents of a county, and the degree to which the individuals tested diverge from a random sample of that population. Depending on the sampling location and time, people who are present to be sampled may be at higher or lower risk of COVID-19 than average (Fig. 1). Important populations, including those in congregate settings (e.g., nursing homes, prisons), are often excluded. For example, persons residing in long term care facilities (LTCFs) may be over- or underrepresented in serosurveys $[10,11]$, which depending on their seroprevalence can produce an over or underestimate of true seroprevalence in the population. If persons in LTCFs are not sampled, this can result in a lower seroprevalence estimate for older individuals [11]. For example, the authors of the New York State serosurvey, which recruited a convenience sample at grocery stores across the state, acknowledge that enrollment disproportionately excluded persons from vulnerable groups who may be more likely to self-isolate at home; individuals who died from or were hospitalized or housebound with COVID-19 infection; and individuals living in LTCFs [12]. If researchers hope to generalize inferences to specific risk groups, they must ensure these groups are included in the survey. Once the population of interest has been clearly defined, they should endeavor to randomly recruit individuals from the population of interest and upweight under-sampled groups. Standardization or inverse-probability-of-sampling weighting can mitigate this type of bias, but only if all relevant predictors of seropositivity are included in the correction [13].

Biases may arise from nonrandom willingness to participate in a survey, even if a random sample of the population is approached to participate. A serosurvey that successfully reaches the population of interest may still suffer from volunteer bias in who participates in testing. This can bias estimates in either direction. Estimates of seroprevalence will be too high if individuals are more likely to accept testing because they think they have been exposed to SARS-CoV-2. On the other hand, a downward bias will occur if individuals accept testing because they are overcautious or if exposed individuals avoid testing because they do not want a positive test result (Fig. 1). In the study design, volunteer bias 


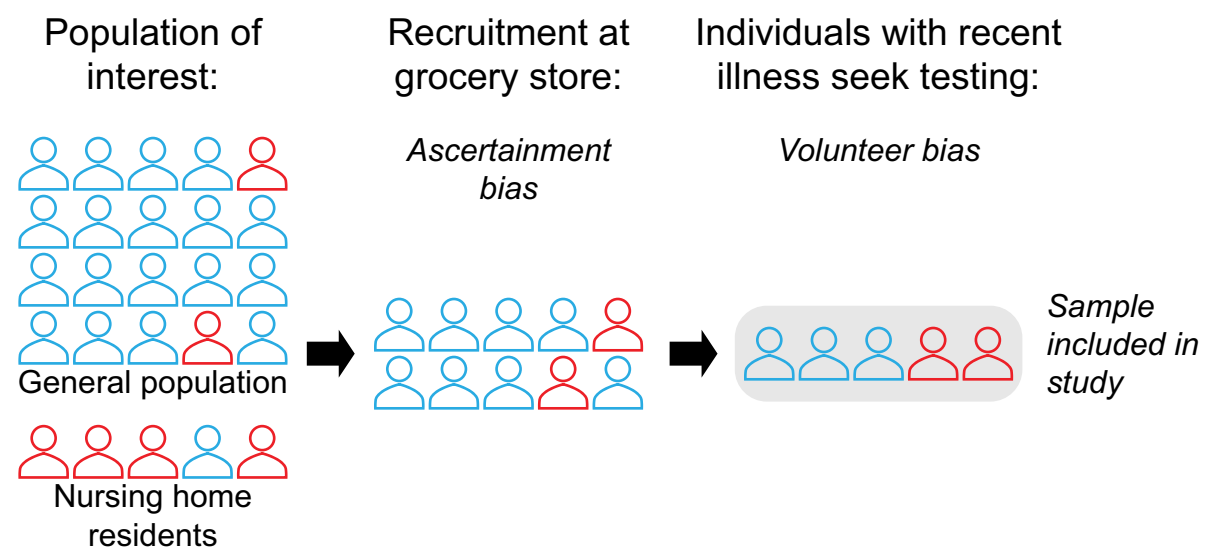

Fig. 1 Schematic showing recruitment-based biases in a hypothetical serosurvey. This figure shows a hypothetical serosurvey that aims to measure the underlying seroprevalence in the entire population of a geographic region and performs recruitment among shoppers at a grocery store. Outline color represents prior SARS-CoV-2 infection status (red for prior infection, blue for no prior infection). Ascertainment bias occurs because (1) individuals recruited at the grocery store are likely at slightly higher risk of COVID-19 than average

can be reduced by sampling from a pre-established cohort with high rates of participation. At least some demographic information should be collected on those who do and do not consent to testing, in order to assess aspects of how representative the consenting population is of those approached. If predictors of non-response are collected, estimates can be corrected in the analysis stage, for example through inverse probability weighting. Serosurveys may want to ask subjects "Do you think you've had COVID-19 previously?", or collect data on symptoms to assess whether volunteer bias is occurring in their sample. Lastly, one innovative approach [14] proposes splitting the survey group into subsets and giving each subset an increasing incentive (for example, money) for participation, enabling researchers to construct a statistical model to predict how seroprevalence would change if everyone participated.

False negative serologic tests, if not properly accounted for, can underestimate seroprevalence, while false positive tests, if not properly accounted for, can overestimate it-the latter problem being most serious near the start of the epidemic. Tests for SARS-CoV-2 antibodies are imperfect. Test performance is described by the test's sensitivity, which is the ability to identify those who have SARS-CoV-2-specific antibodies, and specificity, which is the ability to identify those who do not have such antibodies. Unless adjusted for in the analysis, the use of imperfectly sensitive tests will underestimate the cumulative incidence of past infections due to the presence of infections not detected by the test (Fig. 2). Conversely, a test with imperfect specificity will incorrectly classify individuals without antibodies as positive, resulting in an overestimate of cumulative incidence if (since individuals who are isolated at home and rarely grocery shop are less likely to be sampled), and (2) nursing home residents and other populations in congregate settings are excluded from the sample. Among individuals present at the grocery store, volunteer bias occurs when individuals who believe they have been infected participate in order to receive testing. Meanwhile, individuals who do not think they have been infected may avoid testing

not adjusted for in the analysis (Fig. 2). When a disease is rare, such as COVID-19 early in the pandemic or in areas with low transmission, high test specificity is needed to accurately measure the seroprevalence. For example, the Santa Clara study, which claimed that there were 50-85 times more COVID-19 cases in Santa Clara than previously identified, found 50 individuals positive for antibodies out of 3330 tested [15]; however, the specificity of the test used in that study was uncertain, and a test with $98.5 \%$ specificity would be expected to generate 50 false positives on average in that sample if no one had antibodies.

Adjustments for test sensitivity and specificity should be done with care, accounting for the often small numbers of validation samples and possible differences between the populations in which the tests were validated and the study population. While most serologic studies do adjust for the test sensitivity and specificity using available estimates for each test, the values of sensitivity and specificity in the study population may be different than in the population used for evaluating test performance, which is commonly made up of hospitalized patients [3]. In particular, sensitivity is often lower for individuals with lower antibody titers. Therefore rates of false negatives are expected to be higher among individuals with less severe disease $[8,16]$, such as younger individuals $[17,18]$, individuals who were recently infected and have not yet mounted an antibody response [8,9], or individuals who were infected long before testing, as antibody titers wane over the weeks and months after infection $[8,9]$. For instance, if a seroprevalence estimate among a college student population (where test sensitivity is likely lower) is corrected using a measurement of test sensitivity 
Imperfect test

characteristic
Direction of
resulting bias
Factors affecting

test characteristic

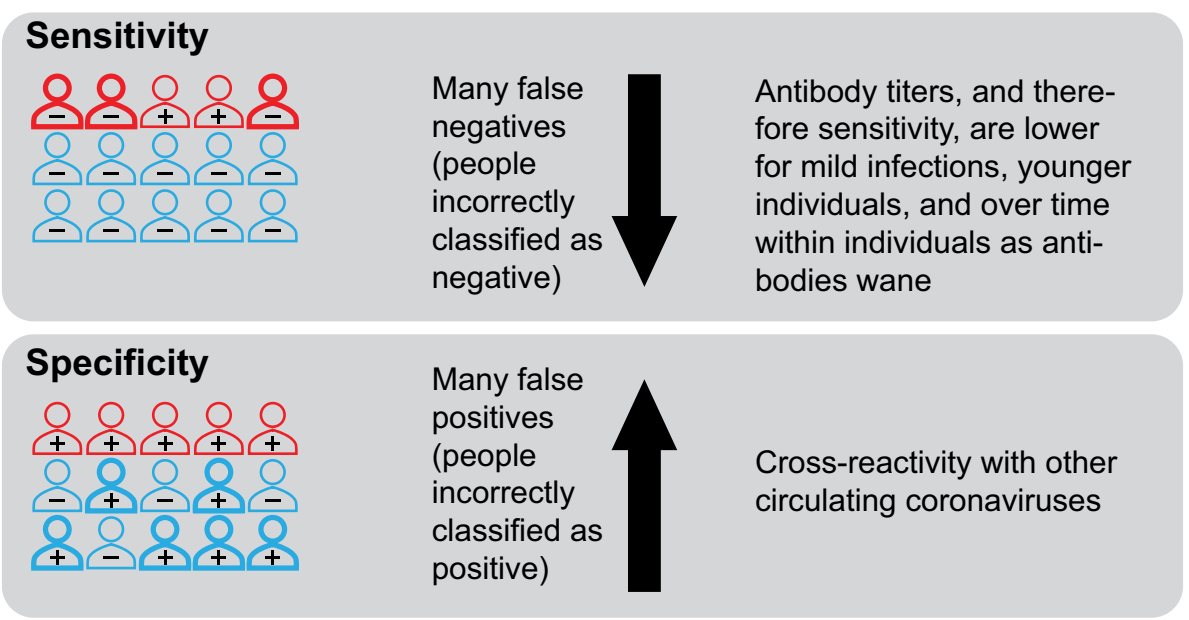

Fig. 2 Biases due to misclassification by SARS-CoV-2 antibody tests. The sensitivity of a SARS-CoV-2 antibody test is the probability the test is positive given an individual has been infected with the virus, while the specificity is the probability of a negative test given an individual has not been infected with SARS-CoV-2. Test performance is imperfect; low sensitivity can result in an estimate of cumulative incidence that is too low (as individuals with prior infection are mis- classified as negative), and low specificity can result in an estimate of cumulative incidence that is too high (as individuals without prior infection are misclassified as positive). Outline color represents prior SARS-CoV-2 infection status (red for prior infection, blue for no prior infection). The annotation ("+" or "-") indicates the result of a test for SARS-CoV-2 antibodies. Bold outlines indicate individuals who are misclassified by the test from older, sicker individuals in validation data then the adjusted estimate will be too low, and vice versa (see [3] for illustrations of this bias through simulation).

As mentioned above, seroprevalence may underestimate cumulative incidence if some individuals who initially have antibody levels sufficient to test positive on a serologic test have waning levels that drop below the threshold for positivity, a phenomenon sometimes called "seroreversion". Low antibody values occur as antibodies are increasing and as they are declining; however, the increase is fast compared to the decline $[9,19]$, so most individuals with low titers will be those on the decline, except perhaps in a very rapidly growing epidemic, where there will be many very recent infections (e.g., [20] but with antibody titers instead of viral load). Antibodies to seasonal coronaviruses have been shown to decline substantially within a period of a few months to a year [21]. Recent evidence points to the similar disappearance of antibodies to some components of SARS-CoV-2 when data is presented as the percent above a threshold defined as positive [22]. Low sensitivity due to waning antibodies is problematic when using seropositivity as a proxy for the cumulative incidence of infection in a population; for example, the observed temporal decline in seroprevalence in several studies [10,23], if more than is explainable by sampling variation, likely indicates waning of antibody titers, as the cumulative incidence of infection cannot decrease with time in a closed population. Much remains to be learned (from seroprotection studies) about the nature and duration of protection following infection, so we do not take a position here on whether cumulative incidence and immunity are the same, but we note that it is biologically possible for an individual to be at least partially immune to infection and/or disease due to $\mathrm{T}$ cell and $\mathrm{B}$ cell memory despite low antibody titers [24-27]. By presenting full distributions of quantitative (e.g., ELISA) values, instead of reporting the percent positive above a threshold, seroprevalence studies can preserve the data for reanalysis as our understanding of antibody kinetics improves.

Solutions to misclassification include prioritizing high specificity when seroprevalence is low, and high sensitivity when seroprevalence is high (Fig. 3) either through test selection or by using multiple independent tests (e.g., [11]). While estimates of seroprevalence at the population level can potentially be corrected for imperfect test characteristics, this may not remove all sources of bias. As discussed above, bias can remain if, for example, the estimates of test characteristics are obtained from "gold standard" positives and negatives with a different distribution of antibody levels than the true positives and negatives, respectively, in the study population [3]. Additionally, there is uncertainty in the measurement of test specificity and sensitivity, especially for newly developed SARS-CoV-2 antibody tests for which validation datasets may be small. Adjustment using point estimates instead of the full ranges of plausible sensitivity and specificity values underestimates the true uncertainty in seroprevalence estimates. A way to rectify these biases is through the use of a Bayesian 


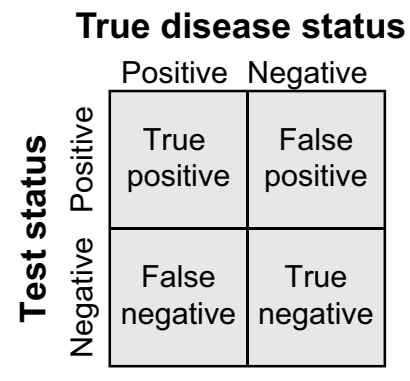

Fig. 3 The relative importance of test sensitivity and specificity depends on the underlying seroprevalence in the study population. The value of a test can be described through the positive predictive value (PPV), which is defined as the probability that an individual truly has been infected with the virus given that they test positive and is calculated as the number of true positives divided by the total number of positive tests. Similarly, the negative predictive value (NPV) is defined as the probability that an individual truly has not

approach to adjust seroprevalence estimates for ranges of values for test characteristics [28].

SUMMARY: an ideal study design for SARS-CoV-2 seroprevalence would:

- Use a sample that is representative of the target population. In particular:

Recruit participants in a way that does not systematically favor those with unusually high or low levels of exposure.

Ask participants and nonparticipants whether they believe they have been infected to detect volunteer bias in a sample.

Consider demographics and other information about participants (and ideally also nonparticipants) to facilitate adjustment of results.

- Use an assay for which sensitivity and specificity estimates are available from a population similar to that being studied in terms of disease severity and timing of infections.

- Report the distribution of quantitative (e.g., ELISA) values and not just the percent positive above a threshold to allow analysis of possible seroreversions.

- Adjust seroprevalence estimates for test characteristics, including uncertainty in the measurements of these characteristics.

\section{Seroprotection}

There is evidence to suggest that prior infection with a coronavirus, including SARS-CoV-2, confers some level of immunity and protection against reinfection with the same been infected with the virus given that they test negative and is calculated as the number of true negatives divided by the total number of negative tests. When the underlying seroprevalence is low, test performance is largely a function of specificity, as the majority of individuals in the population have not been infected, while sensitivity is more important as seroprevalence increases. Note that the negative predictive values for the baseline and low specificity tests are very similar so the curves nearly overlap in the figure

viral species [21, 29-31]. However, the extent and duration of this protection is unknown, and studies are needed to better characterize immunity to this novel virus. While seroprevalence studies focus on one point in time, seroprotection studies are longitudinal, following people over time to evaluate whether seropositivity confers protection against infection compared to seronegativity. In this case, the causal effect of interest is the direct (biological) effect of seropositivity on future infection. A number of biases can arise in these observational studies.

Estimates of the (total) effect of prior infection on (re)infection may be biased toward the null (no protection) if seropositive individuals remain more exposed to infection than seronegative ones (confounding by risk of infection). This scenario occurs when there is a confounder that persists through time, such as residing in a crowded household or being an essential worker, that may predict both the exposure (seropositivity) and the outcome (future infection). For example, people in higher-risk occupations are more likely to become infected at each point in time, meaning they are more likely to be seropositive and also more likely to be reinfected (Fig. 4). These positive associations of the persistent confounder with both the exposure and the outcome create a downward bias, causing seropositivity to appear less protective against (or even harmful for) future infection. Limiting the study to groups with high rates of infection and risk of exposure can mitigate this bias while improving power [5], otherwise this bias can be addressed by adjusting for occupation or other factors associated with risk of infection.

Seroprotection estimates may be biased in either direction if individuals are enrolled at varying phases of their local epidemics or from communities with differently sized outbreaks. People who are enrolled into a seroprotection study 


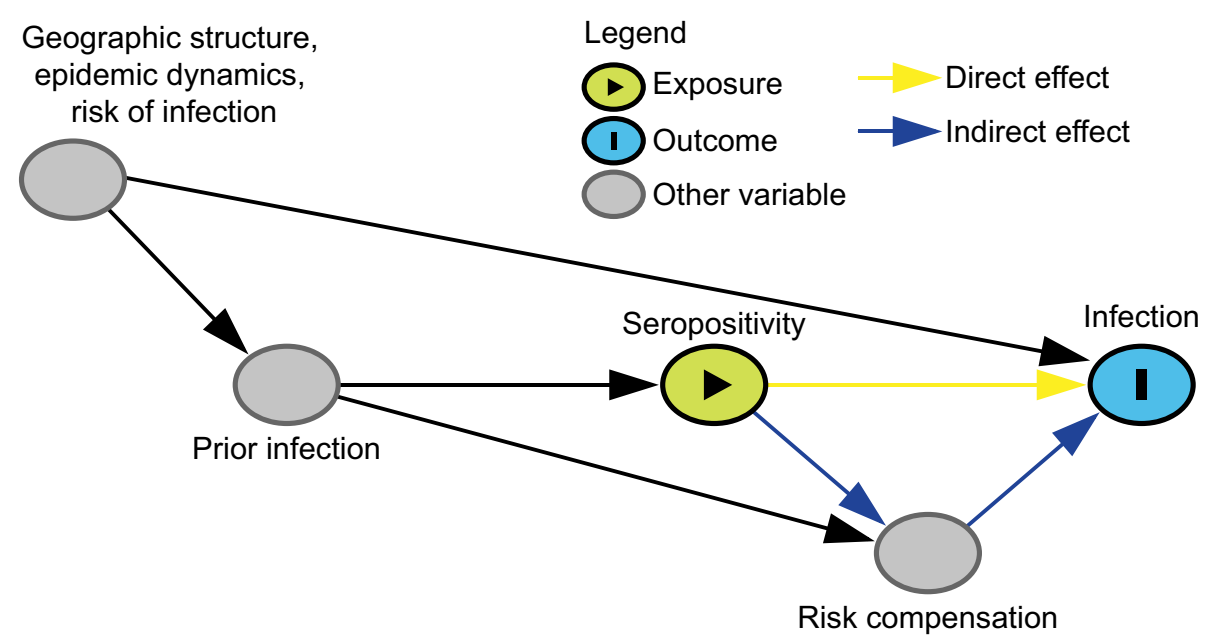

Fig. 4 Directed acyclic graph under the alternative hypothesis showing confounding in the estimation of seroprotection. This figure shows the causal relationship between important variables that influence the infection status of an individual. To analyze the effect of seropositivity on the risk of infection, we would need to adjust for geographic structure, epidemic dynamics, the risk of infection and any other variables that are confounders of this exposure-outcome relationship. The effect of seropositivity on infection risk may be mediated by behavior change (induced by knowledge of serostatus) that affects the risk of infection. Disentangling direct (biological) effects of seropositivity and indirect effects through risk compensa-

in, for example, the early phase of an epidemic are less likely to be seropositive and have a lower daily hazard of infection than those enrolled during the peak of an epidemic. Similarly, study participants enrolled in communities with lower population infection rates are less likely to be seropositive at enrollment and less likely to become infected after enrollment than study participants from communities with higher population infection rates. Adjustments for day of enrollment and community can reduce this bias [5].

Imperfect sensitivity or specificity of serologic tests may result in bias toward the null due to misclassification of exposure status (seropositivity) in seroprotection studies. As noted in the above section on seroprevalence, seropositivity at the population level may imperfectly represent cumulative incidence due to limited sensitivity and specificity and possible changes in these over the course of the antibody response (i.e., declining sensitivity as titers decline). Analogously, at the individual level, which matters for seroprotection studies, seropositivity may be an imperfect representation of an individual's prior infection status. Some of the corrections which are effective at the population level for seroprevalence estimates are not effective at the individual level [32]. Misclassification at the individual level may reduce power as well as cause bias [33].

Increases in risky behavior by those who are seropositive (risk compensation) may increase the risk of reinfection for such individuals, thereby reducing the magnitude of tion is not straight-forward. Geographic structure, epidemic dynamics, and risk of infection are likely or guaranteed confounders of the relationship between seropositivity and future infection. For the purposes of illustrating this particular bias, the directed acyclic graph is drawn under the strong assumption of no additional unmeasured confounding; however, a study of seroprotection, like any observational study, may have other common causes of the exposure (seropositivity) and the outcome (future infection) and it is important to think carefully about additional confounders given unique study settings and designs

seroprotection by creating an indirect effect through which prior infection/seropositivity increases the risk of infection. While this will not bias estimates of the total effect of seroprotection, it will make estimating the direct effect of seropositivity on infection (the effect of interest, which does not include indirect effects mediated by changes in behavior) more challenging; without explicit consideration of behavioral changes, the effects that are evaluated in seroprotection studies will be a combination of the direct and indirect effects (Fig. 4). One potential study design to better isolate the direct effect includes restricting the study to seropositive individuals and comparing high vs. low antibody levels (since people do not usually know this value it should not affect behavior). On the other hand, if antibody levels are a function of previous disease severity $[8,16]$ and disease severity in turn affects future behavior, this could create a different bias. Moreover, negative control outcomes [34] (e.g., risk of other respiratory infections such as respiratory syncytial virus) could be considered to assess the magnitude of the effect due to behavior differences between seropositive and seronegative individuals. Assuming that SARS-CoV-2 antibodies do not protect against influenza, differences in the number of cases of influenza between SARS-CoV-2 seropositives and seronegatives may indicate behavioral differences between the two groups. Another option may be to perform a formal mediation analysis, but the interventions defining this analysis must be explicit and plausible [35-37]. 
SUMMARY: an ideal study design for SARS-CoV-2 seroprotection would:

- Explicitly define a causal effect (estimand) of interest, e.g. with respect to a target trial [13].

- Adjust for factors associated with the risk of infection to reduce confounding.

- Control for (in the analysis) or match on (in the study design) time of enrollment and geographic location to mitigate confounding by epidemic dynamics.

- Think carefully about additional confounders given the unique study setting and design.

- Account for, or at least acknowledge, possible bias and/or loss of power due to imperfect sensitivity and specificity of the serologic assay.

- Give thought to the impact of risk compensation among seropositives on the effects estimated in the study.

- Consider the generalizability of the results given the dynamics of the epidemic during the trial.

\section{Infection risk factors}

When a new infectious disease epidemic arises, some of the most important questions are who is most at risk of acquiring infection and, among those most vulnerable to infection, which groups are more likely to face severe illness or death. These questions are especially relevant for COVID-19, as the pandemic has disproportionately affected communities of color, people living in poverty, and other marginalized groups in the United States and internationally [38-41]. Epidemiological studies are crucial for identifying demographic factors (e.g., age, gender, race/ethnicity, disability status, socio-economic status, job type), as well as structural factors (e.g., living and working conditions, literacy, racism, gender inequity) that are associated with the risk of infection in order to inform the allocation of resources and optimize the impact of prevention and treatment interventions [42]. We refer to these as "risk factors" whether they are true causal factors or statistical predictors of infection [43]. Even if we are only looking to identify statistical predictors of infection risk, these studies can still suffer from selection bias due to excluding certain populations or differential testing rates among populations, and from differential misclassification bias due to assuming non-tested individuals are uninfected or by combining test results across test types, timing of tests, and reasons for testing.

If a study considers a selected group of individuals who are tested to ascertain infection risk factors, selection bias can play a role (in either direction) if the risk factor of interest is related to the likelihood of hospitalization/death, and hospitalization/death also affects the likelihood of being tested. For example, if serologic testing is performed in the community (i.e., among non-hospitalized individuals at some point in time during the outbreak), individuals who are currently hospitalized for severe infection or have died because of COVID-19 will not be included in this cohort. If infected individuals with a certain risk factor, such as a comorbidity, are more likely to experience severe disease and become hospitalized or die, there may be a negative correlation between that risk factor and infection in the study because those individuals are underrepresented in the study (Fig. 5a). This will lead to a spurious correlation under the null hypothesis and an attenuated effect estimate if the factor of interest is a true risk factor for infection. This bias can be limited by including individuals who are hospitalized at the time of testing in the sample or accounting separately for their exclusion, as could also be done for individuals who have died due to COVID-19.

Studies of infection risk factors that include selected groups of individuals who are tested may also suffer from another selection bias if there are differential testing rates or differences in testing criteria between the groups. For instance, testing for SARS-CoV-2 in many parts of the world has often been limited to suspected cases with symptoms. Thus, symptomatic people are more likely to seek testing or may be more likely to be identified as a contact and tested compared to individuals with atypical or no symptoms. If the risk factor of interest affects the likelihood of such symptoms, then selection bias may occur (Fig. 5b). As another example, suppose a study aimed to investigate the relationship between gender and COVID-19 among tested individuals; women, especially younger women, are more likely to seek medical care than men and therefore are more likely to be tested [44]. Men who receive a test may primarily be those with more severe symptoms or with known exposure to SARS-CoV-2. This selection bias will cause a spurious negative correlation between female gender and infection among those who are tested and enrolled in the study. The bias can be avoided by (1) testing all members of a cohort regardless of symptoms or (2) stratifying on the reason for testing, such as respiratory symptoms [45], although this can limit the generalizability of the results.

In studies that use confirmed cases among the population as the outcome of interest, including both tested and untested individuals, differential misclassification bias can occur if untested individuals are assumed to be uninfected. To avoid the selection bias described in the preceding paragraph, studies may include individuals who were not tested for infection and assume they were never infected. Due to this assumption, misclassification of the outcome (infection status) will be much higher among individuals who were not tested (because of lack of symptoms and/or not seeking medical care) compared to tested individuals. This design is unlikely in a formal epidemiological study, but could occur in an ad hoc analysis of case counts. However, 
Fig. 5 Directed acyclic graph under the null hypothesis showing the possible structure of selection bias due to a exclusion from testing and $\mathbf{b}$ differential likelihood of testing. Under the null hypothesis (of no effect of Risk Factor A on COVID-19 infection) selection bias can be in either direction depending on whether Risk Factor A increases or decreases the likelihood of (a) severe disease or (b) symptoms among infected individuals. The figures are simplified to illustrate these particular biases so make the strong assumption of no additional unmeasured confounding (i.e., no common causes of any two variables in the figure) a

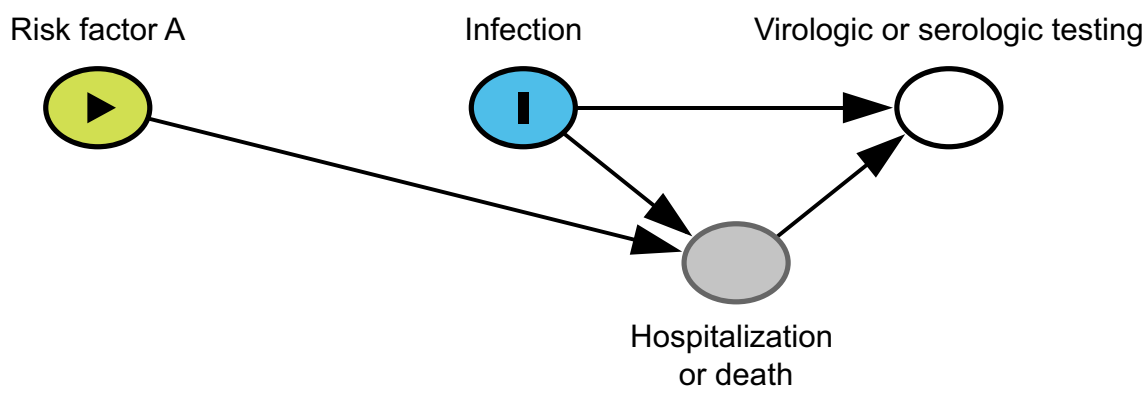

b

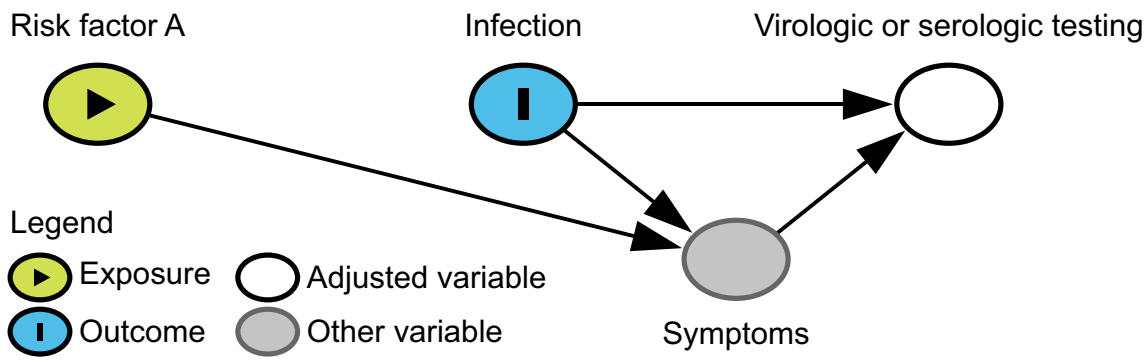

this approach can cause differential misclassification bias, in which risk factors for testing appear as risk factors for COVID-19 [46, 47]. Differential misclassification bias will occur if the risk factor increases (or decreases) the chance of testing through a causal pathway unrelated to the probability of infection (Fig. 6a). For instance, suppose a study aims to investigate whether pregnancy is a risk factor for SARS-CoV-2 infection. In some healthcare facilities and municipalities, pregnant women are routinely screened for SARS-CoV-2 infection upon admission for delivery [48]. In this case, pregnancy will be associated with a higher likelihood of being tested and, therefore, a lower likelihood of being misclassified as uninfected due to not receiving testing. This will induce a spurious positive correlation between pregnancy (or factors correlated with pregnancy, such as gender and age) and infection. Similar differential misclassification by whether an individual is tested may also occur with age, as younger individuals are tested less frequently than older individuals, especially in the early phases of the pandemic [49, 50].

Differential misclassification bias can also arise if testing is performed at different time points, both virologic and serologic testing are used in the outcome measure, or the presence of symptoms affects test performance [51] (Fig. 6b). For example, suppose a study aims to investigate the relationship between age and SARS-CoV-2 infection risk. Virologic (e.g., PCR) testing of children early in the outbreak was less frequent compared to adults because disease in children is relatively mild [49, 50]. Therefore, children may seem less affected in studies that include only virologic testing. On the other hand, given that serologic testing only became available later [52], children may be more likely to be tested with serologic tests compared to adults in a study that combines both serologic and virologic testing. If the total positivity rates for children and adults were compared, combining both test types, children would appear to have a higher risk of infection under the null because they were more likely to undergo serologic testing (a cumulative vs. point-in-time measure) and it occurred at a later time point. Even in studies using only serologic testing, if children were on average tested later than adults, they would have had more opportunities to become infected, which may still induce a correlation. To prevent this bias, studies of infection risk factors should avoid comparison of serology results from one group with virologic testing from another group, or comparison of combined serologic and virologic testing between groups. Additionally, this bias can be reduced in analysis by adjusting for (e.g., through stratification, matching, or control for) the type and timing (with respect to epidemic time) of the test.

SUMMARY: an ideal study design of SARS-CoV-2 infection risk factors would:

- Test all enrollees using the same test type at a fixed time point or set of fixed time points; and

- Include individuals who were hospitalized or died due to COVID-19 in the enrolled population or account for their exclusion; and

- Enroll individuals who have been randomly selected for testing through an infection surveillance program or some other mechanism; or

- Include only tested individuals (limiting the generalizability) and stratify on or otherwise adjust for the type, timing, and reason for receiving the test, as well as 


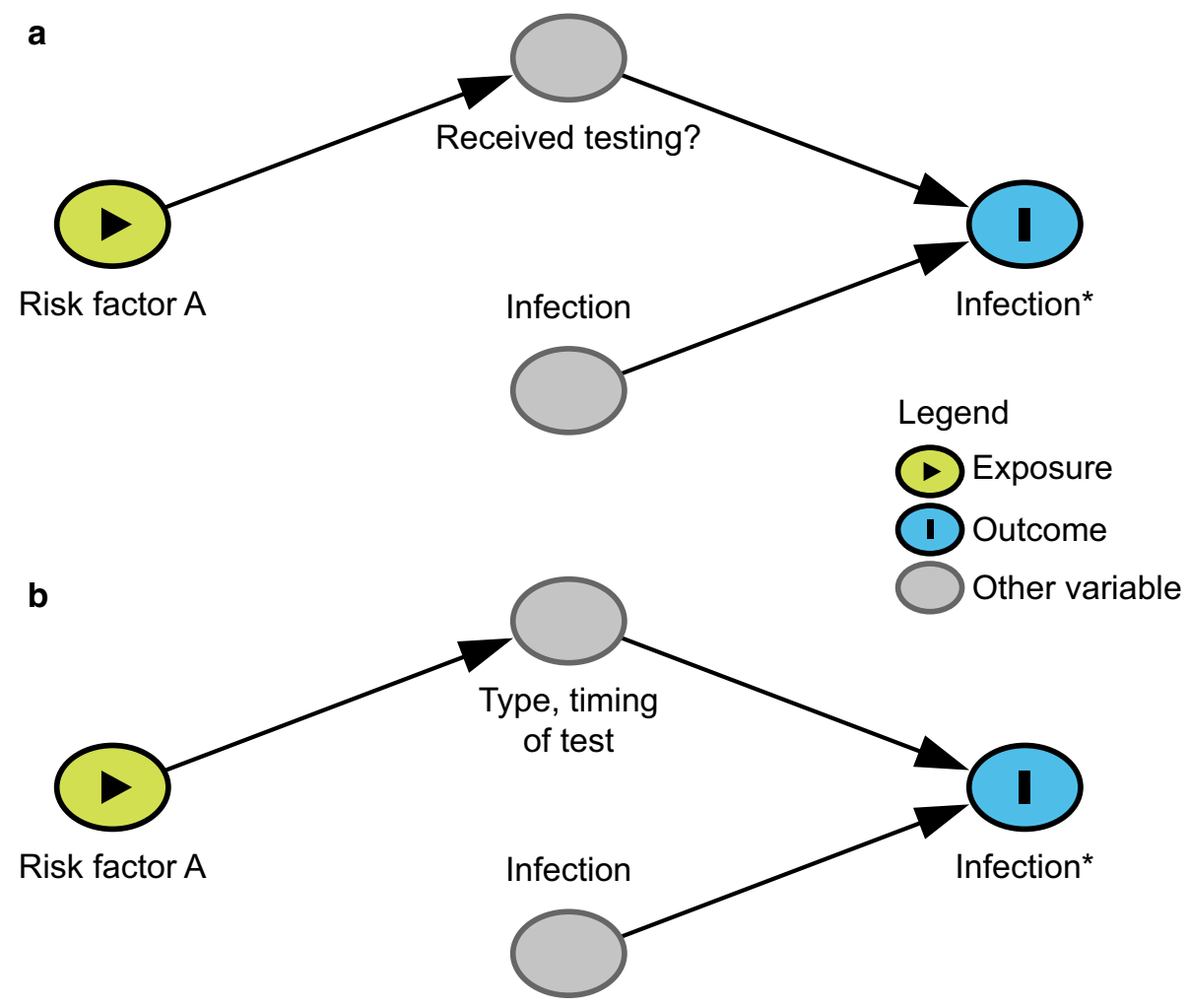

Fig. 6 Directed acyclic graph under the null hypothesis showing differential misclassification by a whether an individual is tested and b the timing or type of test. A study is trying to determine the relationship between Risk Factor A and observed infection status (Infection*), where observed infection status is a proxy for the variable of interest, true infection status (Infection). If (a) Risk Factor A influences whether someone is tested and all non-tested individuals are

account for individuals not tested due to hospitalization or death.

\section{Secondary attack rate estimation}

We first define and differentiate the terms "infectiousness" and "secondary attack rate" (SAR). We define the term infectiousness as the probability that an infected host transmits the infection to a susceptible person during some well-defined type of contact or interaction. Infectiousness depends on factors associated with the pathogen (e.g., quantity shed, transmission-favoring mutations), host factors in the infected person (e.g., age, symptoms, severity of illness, aerosol generation), and host factors in the susceptible individual (e.g., age, health status). With the accumulation of viral genomes, recent studies have reported evidence of mutations that increase transmissibility. For example, the famous D614G mutation is associated with higher viral load and infection of younger hosts [53], and the SARS-CoV-2 VOC-202012/01 strain with the N501Y mutation has been assumed to be uninfected or (b) Risk Factor A affects the type of test and timing of testing conducted then under the null hypothesis of no effect of Risk Factor A on COVID-19 rates misclassification can cause upward or downward bias. The figures are simplified to illustrate these particular biases, and therefore make the strong assumption of no additional unmeasured confounding (i.e., no common causes of any two variables in the figure)

estimated to have a $\mathrm{R}_{0} 1.75$ times higher than the $501 \mathrm{~N}$ strain [54]. However, without long-term observation and rich sequencing data we cannot know the relative transmissibility of the virus strains observed in different studies. Therefore, we will limit these discussions to factors relevant for control measures - that is, we assume that the biological features of the virus do not change when comparing different studies. Under this assumption, we expect that infectiousness will differ for different kinds of contact (e.g., being in proximity vs. touching vs. kissing), with different precautions (e.g., with or without mask wearing), and in different environments (e.g., indoor vs. outdoor, degree of ventilation). In an ideal world, infectiousness would be measured by the "susceptible-exposure attack rate", which is the proportion of exposures per susceptible contact leading to a transmission event - that is, infectiousness per contact, with contact being precisely defined [55]. Since exposures themselves are rarely observed, the SAR is often used as a proxy measure for infectiousness or susceptibility.

The SAR is the proportion of susceptible individuals who become infected within a group of susceptible 
contacts of a primary (index) case within a given time period [56]. The denominator is the total number of individuals contacted in a particular setting (a particular study may but often does not assess the susceptibility of these individuals at baseline). The numerator is the number of infected secondary cases among those contacts. Contact tracing studies identify and collect information about the index case(s) [usually defined as the first identified infected individual(s)] as well as the close contacts, who are followed to observe the outcome of the exposure. SAR estimation in a particular setting, such as a household or school, can help identify the role of different social interactions, environmental factors, characteristics of the index case, and susceptibility of contacts, which can inform effective strategies to prevent onward transmission. However, biases in study design or data analysis can give rise to inaccurate SAR estimation leading to the mischaracterization of infectiousness or susceptibility. Here we summarize biases that result in inaccurate estimation of the SAR, provide some examples from current studies, and outline recommendations that should be considered to provide accurate estimates and interpretations of infectiousness and susceptibility.

Biases in estimating the SAR can be introduced through two key mechanisms: misclassification of the index case(s) (section "Secondary attack rate estimation: misclassification of the index case(s)", Figs. 7 and 8, Fig. S1) and

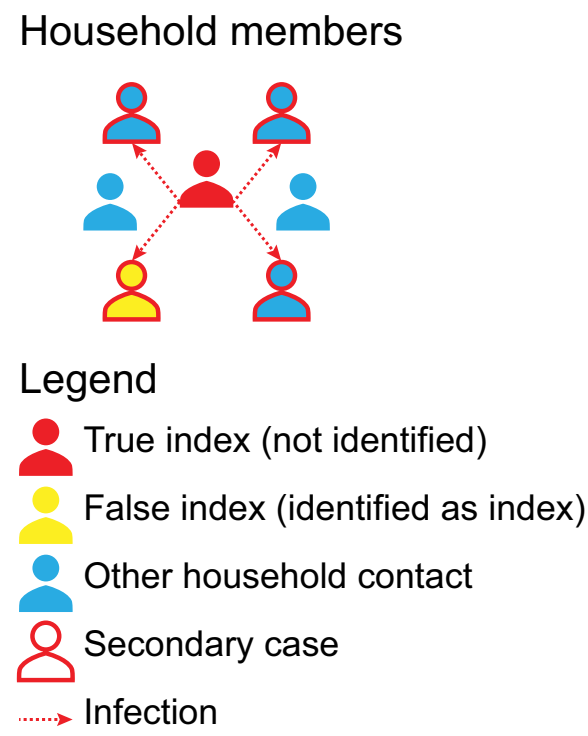

Fig. 7 Illustration of index case misclassification where the index and secondary cases are misclassified in a household scenario. In this scenario (top left), each individual has close contacts with every other household member, and the red arrows indicate infections transmitted by the true index case (red individual) to other household members. The true SAR is shown in the top right; the infected contacts of the true index are in the numerator and all contacts are in the denominator. Index case misclassification can happen if one of the secondary misclassification of close contacts (section "Secondary attack rate estimation: misclassification of close contacts", Fig. 9).

\section{Secondary attack rate estimation: misclassification of the index case(s)}

Misclassification of the index case(s) can happen in two ways: a non-primary (i.e., secondary or tertiary) case is falsely identified as the index case (Fig. 7, Fig. S1) or only one index case is identified when in fact multiple index cases are present (Fig. 8).

Identifying a non-primary case as the index case may bias SAR estimates up, down, or create no bias in some household settings. In an outbreak investigation or a household contact tracing study, a secondary case that has more obvious clinical symptoms or epidemiological characteristics (i.e., imported vs. local cases) relative to the true index case may falsely be classified as the index case. For COVID-19, it is possible for an index case to develop symptoms later than the secondary cases [57]; therefore, studies that define the index as the first identified case in a cluster, especially in household context $[58,59]$, are prone to this error, which could bias the SAR downward or cause no bias (Fig. 7). For scenarios outside the household, the direction of the bias depends on the relative number of contacts from the true index case and the misclassified one (Fig.

True SAR

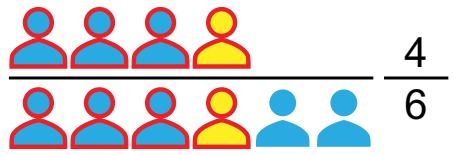

Biased SAR
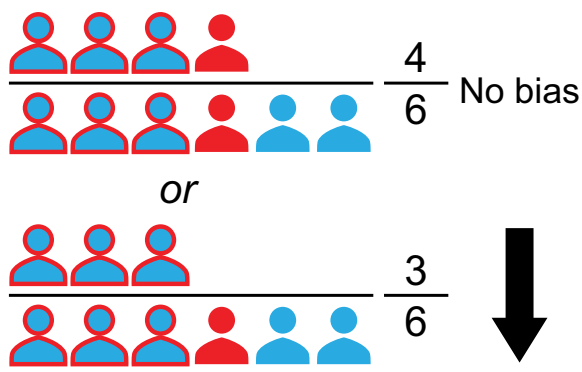

cases of the index is falsely identified as the index case (yellow individual). This may cause no bias in the estimation of the SAR value; however, the interpretation of this SAR may be incorrect because we mistakenly attribute the SAR to the false index case, who may have different characteristics, such as age, from the true index case. It can also introduce downward bias if the true index is no longer detected by PCR by the time they are tested (bottom right) 


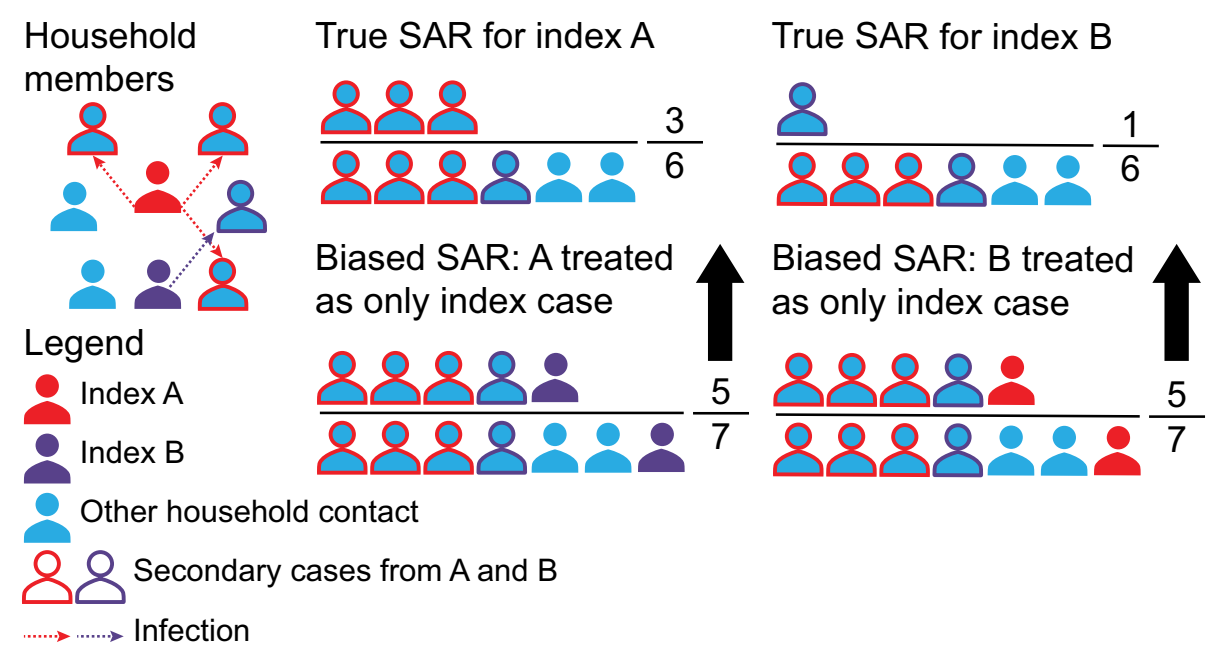

Fig. 8 Illustration of index case misclassification when multiple index cases are present but only one is identified as the index case. As shown in the top left, two index cases (red and purple individuals) acquired the infection and transmitted it (red and purple arrows) to other household members. As members of a household are often considered to all be in contact with one another, we cannot distin- guish who truly infected whom. The true SARs for each index case are shown in the top. The numerator consists of infected contacts and the denominator consists of all contacts. Upward bias in the SAR can be introduced by falsely attributing all infections, including the other index case, to one of the two index cases

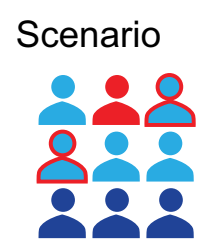

Legend

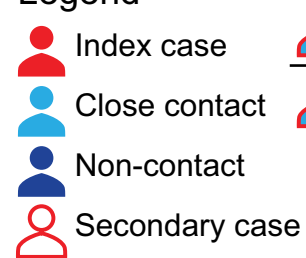

True secondary attack rate

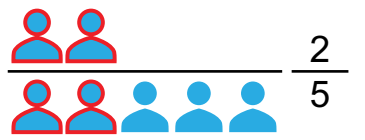

Biased SAR: Misclassification of contact type

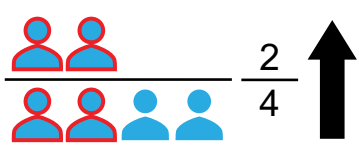

or

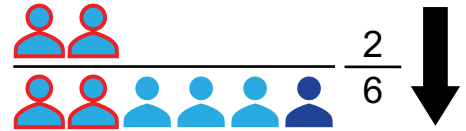

Biased SAR: Misclassification of contact infection status
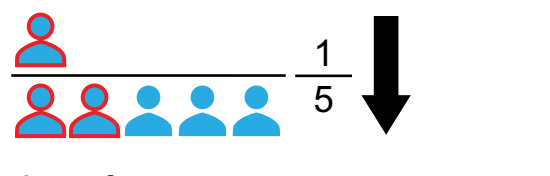

Fig. 9 Illustration of misclassification of contact type and contact infection status. As shown in the top left, an infected individual infects some of their close contacts. The true SAR is represented in the top middle; the infected contacts are in the numerator and all close contacts are in the denominator. Bias due to misclassification of contact type can go in both directions. Bias is in the upward direction

S1). Epidemiological history can cause an analogous bias; imported cases may be more likely to be identified as index cases than local community cases, especially at a relatively early stage of an outbreak when importation of cases from the epidemic center and local transmissions are both happening simultaneously. For example, identified asymptomatic cases during the third wave in Hong Kong in July and August 2020 were more likely to be imported, which may indicate different screening and testing practices for travelers 
causes the SAR to be biased upwards as the secondary cases from B and perhaps even B themselves will be attributed as secondary cases of A. This is most likely in settings with dynamic populations where the source of infection is unclear (i.e., gyms, bars, nursing homes, or gathering events) and multiple index cases may be infecting people at the same time [61, 62]. One example of this bias can be seen in a study from South Korea [58] in which the initial report suggested that the SAR of index cases aged 10-19 years was significantly higher than for index cases aged 20-29, 30-39 and 40-49 years. However, there was a great deal of uncertainty about the true index case in the 10-19 year group due to common sources of exposure with other family members. In a re-analysis [63] of this data, the authors removed household members who potentially shared a common source of exposure with the pediatric cases, resulting in a much lower SAR for the 10-19 year group. Another example is a nursing home outbreak investigation from the Netherlands, where a church service was initially thought to be the source of the outbreak; however, genome sequencing showed multiple clusters in the viral genomes, suggesting multiple introductions to the nursing home [62].

Index case misclassification can be minimized through rigorous follow-up of the selected study population, using frequent and standardized testing, symptom monitoring, and daily contact diaries to track potential infections and index cases. Furthermore, viral genomic analyses, combined with information on contacts, have the potential to more accurately reconstruct transmission pairs or chains of transmission, and further reduce bias in the identification of index cases $[64,65]$. In addition, the chain-binomial model can also be used to avoid the misclassification of secondary or tertiary cases as index cases, especially in household settings [66]. An ideal but less practical solution, particularly early in an outbreak, is to conduct a prospective cohort study, either a household study or another population-based study, in which subjects are enrolled before infection and followed up over time.

\section{Secondary attack rate estimation: misclassification of close contacts}

Imprecise definitions of "close contact" can complicate estimation and interpretation of the SAR. Ambiguity or bias due to misclassification of close contacts is a common problem in contact tracing studies.

Misclassification of close-contact identification is often related to how the study defines and recruits close contacts, which directly impacts the denominator of the SAR estimate (Fig. 9). If the total number of contacts is not fully documented due to an unclear definition of close contact, it could bias the SAR upward because the closest contacts are more likely to be documented. For example, one study investigated how mask wearing in pre-symptomatic patients can prevent SARS-CoV-2 transmission [67]. In this study, a maximum of ten close contacts of each index case were selected even though the index case may have had more than ten close contacts. This artificial limit would bias the SAR upward for index cases with over ten close contacts. On the other hand, if all contacts including non-close contacts are counted as close contacts, that is, all individuals in the setting rather than well-defined close contacts, it would bias the SAR downward. For example, one study [68] included all employees, family members, and clients of a supermarket over a certain time period, although not all individuals had close contact with the index case. While this study reported separate SARs for different contact groups, the overall SAR reported in this study is not comparable to that of other studies that include only close contacts.

Misclassification of infection status in the close contacts directly affects the numerator of the SAR (Fig. 9). If some secondary infections are missed because contacts are not followed for an adequate duration $[68,69]$ or different outcome ascertainment procedures are used for different groups [70], it would bias the SAR downward. For example, if only contacts with respiratory symptoms are tested then this could bias the SAR downward as seen in a study that recruited 445 close contacts, but only tested the 54 who developed new or worsening symptoms during active symptom monitoring [70]. This means untested contacts were counted as uninfected. A similar but less severe bias occurs when different tests with imperfect sensitivity are used for contacts, which could also bias the SAR downward. Misclassification may also exist when a study uses real-time polymerase chain reaction (RT-PCR) but tests contacts too early or too late after exposure, resulting in a low yield in test positivity (an example under Fig. 10). For example, RT-PCR missed 36\% (95\% CI: 28\%, 44\%) of infected close-contacts, especially among those who were tested in the first few days after exposure [71].

Proposed solutions to avoid misclassification of the identification and infection status of close contacts are to refine study protocols beforehand. For example, a clear close contact definition and a standardized protocol to define and identify all potential close contacts throughout the study can help eliminate misclassification. A standardized and high sensitivity testing plan for all close contacts (ideally, regardless of symptom status), and reasonable follow-up (ideally, at least for the duration of the incubation period, for example, 14 days for COVID-19) to observe the outcome of exposure can provide complete ascertainment of the secondary cases.

\section{Interpreting comparisons of the secondary attack rate to make inference about relative susceptibility or infectiousness}

The previous section cataloged the ways in which various biases could affect SAR estimation. If the SAR can be used 


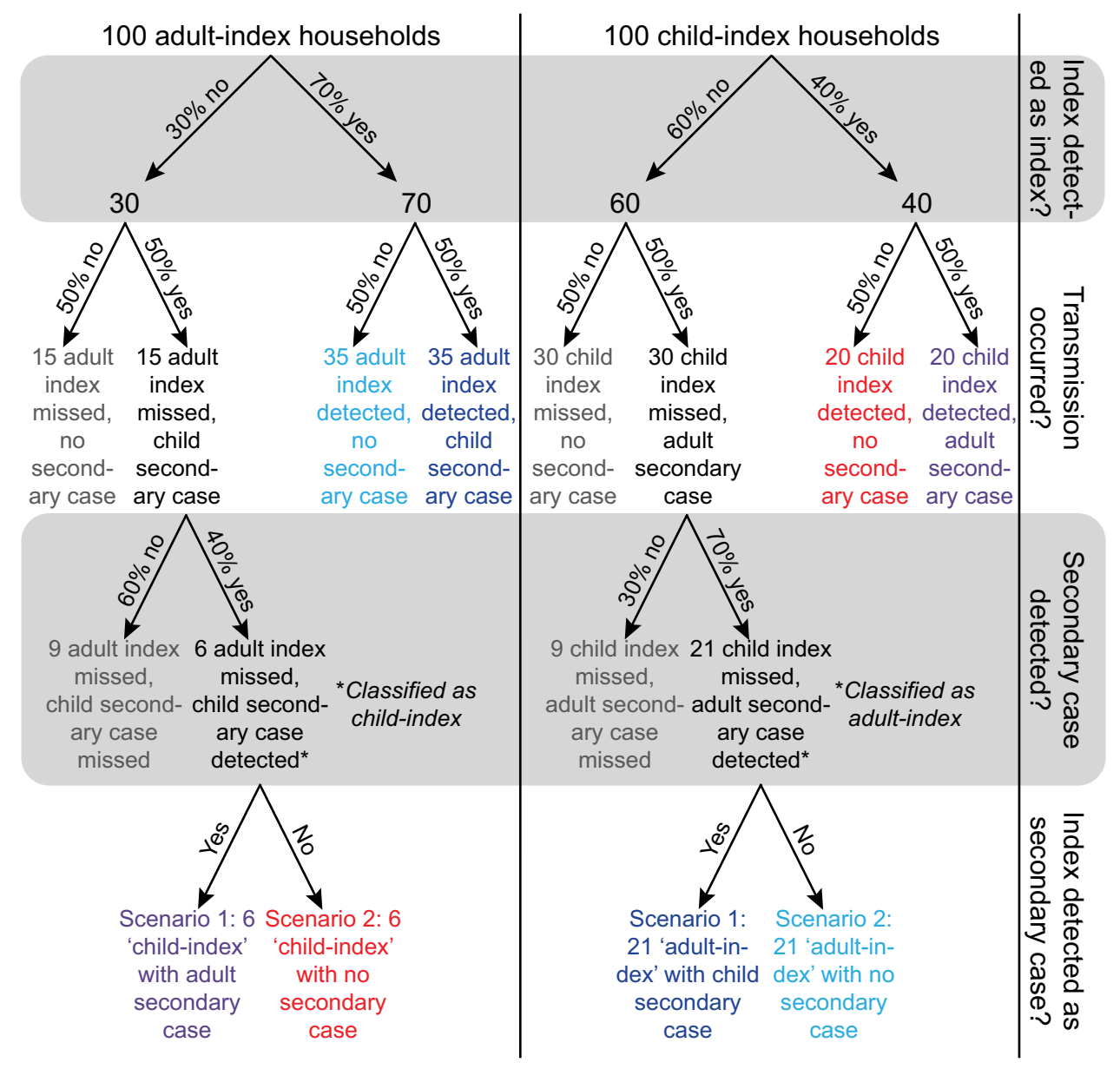

Fig. 10 Illustration of differential detection of infection in adults and children. Flowcharts of infection and detection are presented in the diagram. Households shown in the same colors represent the same results, no matter whether they are true or misclassified. Households in grey remain completely undetected. We consider a simplified example for intergenerational household transmission; all households are composed of one adult and one child, so that the only transmission opportunity is to one individual of the other age category. We use 100 households with an adult index case (left column), and 100 households with a child index (right column). This scenario is drawn under the null hypothesis of equal infectiousness of adults and children, and both age groups transmit the infection half $(50 \%)$ of the time. The only difference between infected adults and children is the probability that they are detected, reflecting differential symptom presentation. We assume that $70 \%$ of adults and $40 \%$ of children are detected (the numbers chosen are illustrative and the key informa-

to estimate infectiousness, then comparing the SAR between index cases can provide information about the association of index case characteristics (e.g., age) with infectiousness. Likewise, SAR comparisons between contacts of different demographic types can be used to infer the relative susceptibility of different types of individuals. However, there are several factors that need to be considered when using the SAR to infer relative susceptibility or infectiousness. Here we discuss factors that influence the interpretation of the tion is that adults are more likely to be detected). Additionally, we assume that testing works perfectly (i.e., all contacts are tested and identified accurately) and no testing is triggered by contacts outside of the household. We consider two scenarios when an index case is missed and their secondary case is detected. In both scenarios the secondary case is falsely considered to be the index case and the true index is tested as a potential secondary case. In scenario 1 , the true index can still be detected and will falsely be considered a secondary case of the false index. In scenario 2, the true index can no longer be detected and the false index will be considered to have not infected anyone. The SARs under both scenarios are calculated in Table 1, which shows that the differential detection of infections in adults and children creates a bias that can go in either direction. Under scenario 1 , the SAR is higher for adult indices than for child indices, while for scenario 2 the SAR is higher for child indices than for adult indices

SAR, such as comparing the SAR by characteristics of the index cases or close contacts, contact patterns, and across environmental settings. For demographics, we use adults compared to children as the running example.

If identical biases were present in estimating the SAR for adult and child index cases, we might expect the comparison of infectiousness and/or susceptibility to be unaffected. However, many of the biases depend on, for example, the probability of identifying someone correctly as an index 
case (as discussed previously in section "Secondary attack rate estimation: misclassification of the index case(s)"), and this may differ between adults and children because children with SARS-CoV-2 infection often have milder or no symptoms [72], and thus may be less likely to be classified as the index case for the cluster than adults. Similarly, children may also be less likely to be identified as secondary cases. Thus, these biases in SAR estimation can differentially affect child and adult index cases and/or contacts, and may lead to biases in comparative infectiousness or susceptibility.

One hypothetical scenario we illustrate here shows how this bias is introduced and the corresponding results, where the bias can go in either direction even under the null hypothesis of equal infectiousness (Fig. 10, Table 1). In this example, where all households have only one child and one adult, the infectiousness of children will be underestimated (and their susceptibility will be overestimated) if missed index cases are misclassified as secondary cases (scenario 1), while the opposite bias will occur if missed index cases are not identified as secondary cases (scenario 2), perhaps because they are no longer positive by the time they are tested. As shown in detail in Fig. 10 and Table 1, differential misclassification between adults and children can lead to biases in either direction in the estimation of their relative infectiousness or susceptibility, even when no such differences exist (i.e., the null hypothesis is true, Fig. 10,

Table 1 Calculation of the SAR when there is differential detection of infection in adults and children

\begin{tabular}{|l|c|c|c|}
\hline \multicolumn{3}{|c|}{ Scenario 1: The true index can still be detected and is falsely considered } \\
a secondary case of the false index \\
\hline & $\begin{array}{c}\text { Number of } \\
\text { households with } \\
\text { transmission }\end{array}$ & $\begin{array}{c}\text { Total number } \\
\text { of households }\end{array}$ & SAR \\
\hline $\begin{array}{c}\text { Households classified } \\
\text { as adult index with } \\
\text { child at risk }\end{array}$ & $35+21=56$ & $56+35=91$ & $56 / 91=62 \%$ \\
\hline $\begin{array}{c}\text { Households classified } \\
\text { as child index with } \\
\text { adult at risk }\end{array}$ & $20+6=26$ & $26+20=46$ & $26 / 46=57 \%$ \\
\hline
\end{tabular}

\begin{tabular}{|c|c|c|c|}
\hline \multicolumn{3}{|l|}{$\begin{array}{l}\text { Scenario 2: The true index can no longer be detected and the false } \\
\text { index is not considered to have any secondary cases }\end{array}$} \\
\hline & $\begin{array}{c}\text { Number of } \\
\text { households with } \\
\text { transmission }\end{array}$ & $\begin{array}{c}\text { Total number } \\
\text { of households }\end{array}$ & SAR \\
\hline $\begin{array}{c}\text { Households classified } \\
\text { as adult index with } \\
\text { child at risk }\end{array}$ & 35 & $35+56=91$ & $35 / 91=38 \%$ \\
\hline $\begin{array}{c}\text { Households classified } \\
\text { as child index with } \\
\text { adult at risk }\end{array}$ & 20 & $20+26=46$ & $20 / 46=43 \%$ \\
\hline
\end{tabular}

The tables show the SAR calculation under the two scenarios displayed in Fig. 10. The differential detection of infections in adults and children creates a bias, which can go in either direction. In scenario 1 , adult indices have a higher SAR than child indices. In scenario 2, adult indices have a lower SAR than child indices
Table 1). We use this simple example for illustration; actual studies will typically have a mixture of household structures and may also face the issues described in the next section.

Besides factors related to the identification of index cases and close contacts, attention must be paid to contact patterns, including the duration of contact, contact frequency, and the setting where contact occurs, when using the SAR to make inferences about infectiousness. This includes heterogeneity in contact behaviors, exposure settings, and contact populations. Individuals may infect more secondary cases simply because they have prolonged and closer contacts or riskier behaviors. Higher risks can come from the gathering pattern, such as living together, sleeping in the same room, dining together, or activities such as singing/shouting, and playing board games [73]. For example, spouses have higher attack rates compared to other household contacts with odds ratios of 2.27 (95\% CI: 1.22, 4.22) [74] and 3.66 (95\% CI: $1.28,10.5)$ [75], indicating that the marital status of household contacts should be accounted for in household studies to better disentangle biological from behavioral factors in the infectiousness of household index cases. The setting of the exposure is also important. Different environmental settings, such as indoor vs. outdoor, household vs. non-household [76], environments that tend to generate aerosols (some clinical treatment processes [77]), or poor ventilated settings, may lead to differences in transmission. Suppose there are two types of individuals - type A and type B-who are identical other than the factor being considered. If, all else equal, individuals of type A have prolonged contact with others in a poorly ventilated setting, then type A indices will have more secondary cases than type B indices. Or if type A indices have more contacts in these settings and engage in higher risk activities when the viral load is highest (i.e., -2 to 5 days after symptom onset [78]) and they are more likely to be highly infectious then they may generate more secondary cases (i.e., superspreading events) than type B indices. The third component in contact heterogeneity is the age structure or demographic characteristics of the close contacts. For example, if type A individuals have more contacts with older individuals, then type A indices may end up infecting more people than type B indices, as emerging evidence suggests susceptibility to infection increases somewhat with age [7]. However, studies rarely report the age structure or underlying conditions of close contacts. While this issue can influence studies about infectiousness or susceptibility, it can be easily avoided by collecting relevant information.

Proposed solutions to accurately infer and compare infectiousness include testing stool samples in children because the duration of viral RNA shedding is longer so they are less likely to be misclassified [79, 80]; reporting differences in contact patterns, including activities/behaviors, duration of contact, contact frequency, and contact setting; and 
collecting detailed epidemiological characteristics of close contacts, such as age, gender, and underlying conditions.

SUMMARY: an ideal study design for SARS-CoV-2 secondary attack rate, infectiousness, and susceptibility estimation would:

- Ensure rigorous follow-up of the study population to minimize misclassification of the index case. This could integrate whole-genome sequencing and phylogenetic analysis to improve the identification of the index case(s), introduction of multiple index cases, transmission directions, chains of transmission, and network interactions. Repeat testing that gives information about viral load may also inform inference of the relative probability that different individuals are index cases [81].

- Use a prospective cohort design, such as a household study, in which subjects are enrolled before infection and followed over time. This could involve frequent serial testing, symptom monitoring, and the use of daily contact diaries. In contact tracing studies, stool samples might be an option for testing children to reduce misclassification of child cases and contacts.

- Clearly define "close contact" and use a standardized protocol for identifying all potential close contacts. Have a standardized and highly sensitive testing plan for assessing infection in all close contacts.

- Use a sufficiently long length of follow-up to observe the outcome of exposure (ideally, at least for the duration of the incubation period, for example, 14 days for COVID19) among all close contacts.

- Consider hypotheses about shared exposures and collect information on them (for example, travel together to an infected area) [82] or stratify results in main or sensitivity analyses to exclude possible shared exposures [83].

- Simulate in silico outcome data for contact tracing studies under different plausible infection scenarios to understand the impacts of potential misclassifications of index cases or close contacts.

- Be aware of differences in contact patterns, including the duration of contact, contact frequency, contact setting, and epidemiological characteristics of close contacts, when using the SAR to infer that certain groups, such as adults, have higher biological, per-contact infectiousness or susceptibility than other groups, such as children.

\section{Conclusion}

To assist in the evaluation of a continually expanding body of literature on COVID-19, we have outlined and proposed solutions to common biases that can occur across different types of observational studies of COVID-19, including cross-sectional seroprevalence, longitudinal seroprotection, risk factor studies to inform interventions, studies to estimate the secondary attack rate, and studies that use the secondary attack rate to make inferences about relative infectiousness or susceptibility. Across study designs, we identified issues of interpretation, as well as possible biases due to measurement error, selection bias, confounding, and recruitment of non-representative samples. In particular, we highlighted how studies of seroprevalence are subject to misclassification by antibody tests and the possible recruitment of nonrepresentative samples, while studies of seroprotection may suffer from confounding by geographic structure, epidemic dynamics and risk of infection, and their interpretation may be complicated by risk compensation. Studies of infection risk factors may be prone to biased selection of subjects, resulting from the presence of symptoms or hospitalization/ death status, and differential misclassification of infection status due to testing factors. Lastly studies of the secondary attack rate can be biased due to misclassification of the index case(s), and failure to correctly identify close contacts and determine their infection statuses, while the use of secondary attack rates to make inferences about infectiousness and susceptibility must be performed carefully with awareness of contact patterns. Although each bias is discussed separately in each study design, multiple biases may coexist and need to be examined carefully in real settings. We hope these thorough descriptions of biases can provide a map or checklist of potential biases to assist with both future study design and the critical interpretation of existing study results.

Supplementary Information The online version of this article (https:// doi.org/10.1007/s10654-021-00727-7) contains supplementary material, which is available to authorized users.

Acknowledgements The authors express their gratitude to Ali Ihsan Nergiz for his help identifying and extracting data from studies cited in the "Secondary attack rate (SAR) estimation" section; Shae Gantt, Yonatan Grad, Siyang Xia, and James Hay for their insightful feedback on the project; and the National Institute of Allergy and Infectious Diseases of the National Institutes of Health (award number T32AI007535), the National Institute of General Medical Sciences of the National Institutes of Health (award number U54GM088558), the Morris-Singer Fund, and the National Institutes of Health (cooperative agreement U01 CA261277) for funding.

Author contributions Emma K. Accorsi, Lee Kennedy-Shaffer, Rebecca Kahn, Edward Goldstein, and Marc Lipsitch conceptualized the project. Emma K. Accorsi, Xueting Qiu, Eva Rumpler, Lee Kennedy-Shaffer, Rebecca Kahn, Keya Joshi, Muge Cevik, and Marc Lipsitch wrote the manuscript. Emma K. Accorsi, Eva Rumpler, Lee Kennedy-Shaffer, Rebecca Kahn, and Keya Joshi created manuscript figures. Edward Goldstein, Mats J. Stensrud, Rene Niehus, Muge Cevik, and Marc Lipsitch provided conceptual feedback. Muge Cevik and Marc Lipsitch supervised the research. All authors edited the manuscript and approved the final manuscript.

Funding EKA was supported by the National Institute of Allergy and Infectious Diseases of the National Institutes of Health under 
award number T32AI007535. XQ, EG, and RN were supported by the National Institute of General Medical Sciences of the National Institutes of Health under award number U54GM088558. ER and LKS were supported by the Morris-Singer Fund. ML was supported by the National Institutes of Health under cooperative agreement U01 CA261277, and the Morris-Singer Fund. The content is solely the responsibility of the authors and does not necessarily represent the official views of the National Institutes of Health or the Morris-Singer Fund.

\section{Compliance with ethical standards}

Conflicts of interest ML reports grants from NIH/NIGMS, during the conduct of the study; personal fees from Affinivax, personal fees from Merck, grants and personal fees from Pfizer, grants from PATH Vaccine Solutions, outside the submitted work.

Open Access This article is licensed under a Creative Commons Attribution 4.0 International License, which permits use, sharing, adaptation, distribution and reproduction in any medium or format, as long as you give appropriate credit to the original author(s) and the source, provide a link to the Creative Commons licence, and indicate if changes were made. The images or other third party material in this article are included in the article's Creative Commons licence, unless indicated otherwise in a credit line to the material. If material is not included in the article's Creative Commons licence and your intended use is not permitted by statutory regulation or exceeds the permitted use, you will need to obtain permission directly from the copyright holder. To view a copy of this licence, visit http://creativecommons.org/licenses/by/4.0/.

\section{References}

1. Teixeira da Silva JA, Tsigaris P, Erfanmanesh M. Publishing volumes in major databases related to Covid-19. Scientometrics. 2020;126:831-42.

2. Else H. How a torrent of COVID science changed research publishing-in seven charts. Nature. 2020;588:553.

3. Takahashi S, Greenhouse B, Rodríguez-Barraquer I. Are SARSCoV-2 seroprevalence estimates biased? J Infect Dis. 2020. https ://doi.org/10.1093/infdis/jiaa523.

4. Addetia A, Crawford KH, Dingens A, Zhu H, Roychoudhury P, Huang M-L, et al. Neutralizing antibodies correlate with protection from SARS-CoV-2 in humans during a fishery vessel outbreak with high attack rate. medRxiv. 2020. https://doi. org/10.1101/2020.08.13.20173161.

5. Kahn R, Kennedy-Shaffer L, Grad YH, Robins JM, Lipsitch M. Potential biases arising from epidemic dynamics in observational seroprotection studies. Am J Epidemiol. 2020. https://doi. org/10.1093/aje/kwaa188.

6. Griffith G, Morris TT, Tudball M, Herbert A, Mancano G, Pike L, et al. Collider bias undermines our understanding of COVID-19 disease risk and severity. Epidemiology. medRxiv. 2020. https:// www.medrxiv.org/content/10.1101/2020.05.04.20090506v2.abstr act

7. Goldstein E, Lipsitch M, Cevik M. On the effect of age on the transmission of SARS-CoV-2 in households, schools and the community. J Infect Dis. 2020. https://doi.org/10.1093/infdis/jiaa691.

8. Poland GA, Ovsyannikova IG, Kennedy RB. SARS-CoV-2 immunity: review and applications to phase 3 vaccine candidates. Lancet. 2020;396:1595-606.

9. Sethuraman N, Jeremiah SS, Ryo A. Interpreting diagnostic tests for SARS-CoV-2. JAMA. 2020. https://doi.org/10.1001/ jama.2020.8259.
10. Herzog S, De Bie J, Abrams S, Wouters I, Ekinci E, Patteet L, et al. Seroprevalence of IgG antibodies against SARS coronavirus 2 in Belgium: a prospective cross-sectional nationwide study of residual samples. Epidemiology. medRxiv. 2020. https://www. medrxiv.org/content/10.1101/2020.06.08.20125179v2.abstract

11. Pollán M, Pérez-Gómez B, Pastor-Barriuso R, Oteo J, Hernán MA, Pérez-Olmeda M, et al. Prevalence of SARS-CoV-2 in Spain (ENE-COVID): a nationwide, population-based seroepidemiological study. Lancet. 2020. https://doi.org/10.1016/S0140 -6736(20)31483-5.

12. Rosenberg ES, Tesoriero JM, Rosenthal EM, Chung R, Barranco MA, Styer LM, et al. Cumulative incidence and diagnosis of SARS-CoV-2 infection in New York. Ann Epidemiol. 2020;48(23-9):e4.

13. Hernán MA, Robins JM. Causal inference: what if. Boca Raton: CRC; 2020.

14. Porter E. Don't think you need a coronavirus test? What if I paid you? The New York Times. 2020. https://www.nytim es.com/2020/04/21/business/economy/coronavirus-tests-infec tions.html

15. Bendavid E, Mulaney B, Sood N, Shah S, Ling E, BromleyDulfano R, et al. COVID-19 Antibody Seroprevalence in Santa Clara County, California. Epidemiology. 2020. https://doi. org/10.1101/2020.04.14.20062463.

16. Lynch KL, Whitman JD, Lacanienta NP, Beckerdite EW, Kastner SA, Shy BR, et al. Magnitude and kinetics of anti-SARS-CoV-2 antibody responses and their relationship to disease severity. Infect Dis. (except HIV/AIDS). medRxiv. 2020. https://www. medrxiv.org/content/10.1101/2020.06.03.20121525v1.abstract

17. Lumley SF, Wei J, O’Donnell D, Stoesser NE, Matthews PC, Howarth A, et al. The duration, dynamics and determinants of SARS-CoV-2 antibody responses in individual healthcare workers. Infect Dis. (except HIV/AIDS). medRxiv. 2020. p. CD013652. https://doi.org/10.1101/2020.11.02.20224824

18. Liu Y, Mao B, Liang S, Yang J-W, Lu H-W, Chai Y-H, et al. Association between age and clinical characteristics and outcomes of COVID-19. Eur Respir J. 2020. https://doi.org/10.1183/13993 003.01112-2020.

19. Iyer AS, Jones FK, Nodoushani A, Kelly M, Becker M, Slater $\mathrm{D}$, et al. Dynamics and significance of the antibody response to SARS-CoV-2 infection. medRxiv. 2020. https://doi. org/10.1101/2020.07.18.20155374

20. Hay JA, Kennedy-Shaffer L, Kanjilal S, Lipsitch M, Mina MJ. Estimating epidemiologic dynamics from single cross-sectional viral load distributions. bioRxiv. medRxiv. 2020. https://doi. org/10.1101/2020.10.08.20204222

21. Edridge AWD, Kaczorowska J, Hoste ACR, Bakker M, Klein M, Loens K, et al. Seasonal coronavirus protective immunity is short-lasting. Nat Med. 2020. https://doi.org/10.1038/s4159 1-020-1083-1.

22. Iyer AS, Jones FK, Nodoushani A, Kelly M, Becker M, Slater $\mathrm{D}$, et al. Persistence and decay of human antibody responses to the receptor binding domain of SARS-CoV-2 spike protein in COVID-19 patients. Sci Immunol. 2020. https://doi.org/10.1126/ sciimmunol.abe 0367.

23. Emmenegger M, De Cecco E, Lamparter D, Jacquat RPB, Ebner D, Schneider MM, et al. Early peak and rapid decline of SARS-CoV-2 seroprevalence in a Swiss metropolitan region. Epidemiology. medRxiv. 2020. https://www.medrxiv.org/conte nt/10.1101/2020.05.31.20118554v4.abstract

24. Dan JM, Mateus J, Kato Y, Hastie KM, Yu ED, Faliti CE, et al. Immunological memory to SARS-CoV-2 assessed for up to 8 months after infection. Science. 2021. https://doi.org/10.1126/ science.abf 4063 .

25. Rodda LB, Netland J, Shehata L, Pruner KB, Morawski PA, Thouvenel CD, et al. Functional SARS-CoV-2-specific immune 
memory persists after mild COVID-19. Cell. 2021;184:169-183. e17.

26. Zuo J, Dowell A, Pearce H, Verma K, Long HM, Begum J, et al. Robust SARS-CoV-2-specific T-cell immunity is maintained at 6 months following primary infection. Cold Spring Harbor Laboratory. 2020 [cited 2021 Jan 21]. p. 2020.11.01.362319. https://www. biorxiv.org/content/https://doi.org/10.1101/2020.11.01.36231 9v1 ?ijkey $=032 \mathrm{ae} 87 \mathrm{a} 45 \mathrm{~b} 76 \mathrm{ab} 5 \mathrm{cdefeae} 919 \mathrm{~d} 1 \mathrm{aec} 84 \mathrm{c} 893222$ \&keytype2=tf_ipsecsha

27. Gaebler C, Wang Z, Lorenzi JCC, Muecksch F, Finkin S, Tokuyama M, et al. Evolution of antibody immunity to SARS-CoV-2. Nature. 2021. https://doi.org/10.1038/s41586-021-03207-w.

28. Larremore DB, Fosdick BK, Zhang S, Grad YH. Jointly modeling prevalence, sensitivity and specificity for optimal sample allocation. bioRxiv. 2020 [cited 2020 Oct 20]. p. 2020.05.23.112649. Available from: https://www.biorxiv.org/content/https://doi. org/10.1101/2020.05.23.112649v1

29. Callow KA, Parry HF, Sergeant M, Tyrrell DA. The time course of the immune response to experimental coronavirus infection of man. Epidemiol Infect. 1990;105:435.

30. Reed SE. The behaviour of recent isolates of human respiratory coronavirus in vitro and in volunteers: evidence of heterogeneity among 229E-related strains. J Med Virol. 1984. https://pubme d.ncbi.nlm.nih.gov/6319590/

31. Bao L, Deng W, Gao H, Xiao C, Liu J, Xue J, et al. Lack of Reinfection in Rhesus Macaques Infected with SARS-CoV-2. bioRxiv. 2020. p. 13-990226. Available from: https://www.biorxiv.org/ content/https://doi.org/10.1101/2020.03.13.990226v2

32. Cauchemez S, Horby P, Fox A, Le Quynh M, Thanh LT, Thai $\mathrm{PQ}$, et al. Influenza infection rates, measurement errors and the interpretation of paired serology. PLoS Pathog. 2012;8:e1003061.

33. Copeland KT, Checkoway H, McMichael AJ, Holbrook RH. Bias due to misclassification in the estimation of relative risk. Am J Epidemiol. 1977;105:488-95.

34. Lipsitch M, Tchetgen ET, Cohen T. Negative controls: a tool for detecting confounding and bias in observational studies. Epidemiology. 2010;21:383.

35. Stensrud MJ, Robins JM, Sarvet A, Tchetgen Tchetgen EJ, Young JG. Conditional separable effects. 2020. Available from: http:// arxiv.org/abs/2006.15681

36. Robins JM, Richardson TS, Shpitser I. An interventionist approach to mediation analysis. 2020. Available from: http://arxiv.org/ abs/2008.06019

37. Robins JM, Richardson TS. Alternative graphical causal models and the identification of direct effects. In: Shrout P, Keyes K, editor. Causality and psychopathology: finding the determinants of disorders and their cures. Oxford: Oxford University Press; 2010. pp. 103-58.

38. Kim SJ, Bostwick W. Social Vulnerability and Racial Inequality in COVID-19 Deaths in Chicago. Health Educ Behav. 2020;47:509-13.

39. Shadmi E, Chen Y, Dourado I, Faran-Perach I, Furler J, Hangoma $\mathrm{P}$, et al. Health equity and COVID-19: global perspectives. Int J Equity Health. 2020;19:104.

40. Patel JA, Nielsen FBH, Badiani AA, Assi S, Unadkat VA, Patel B, et al. Poverty, inequality and COVID-19: the forgotten vulnerable. Public Health. 2020;183:110-1.

41. Hooper MW, Nápoles AM, Pérez-Stable EJ. COVID-19 and racial/ ethnic disparities. JAMA Am Med Assoc. 2020;323:2466-7.

42. Cevik M, Marcus JL, Buckee C, Smith TC. SARS-CoV-2 transmission dynamics should inform policy. Clin Infect Dis. 2020. https://doi.org/10.1093/cid/ciaa1442.

43. Huitfeldt A. Is caviar a risk factor for being a millionaire? BMJ. 2016;355. https://www.bmj.com/bmj/section-pdf/935674?path=/ bmj/355/8086/Food_for_thought.full.pdf
44. Number of coronavirus (COVID-19) cases in England as of July 30, 2020, by age and gender. Statista. https://www.statista.com/ statistics/1115083/coronavirus-cases-in-england-by-age-andgender/

45. de Lusignan S, Dorward J, Correa A, Jones N, Akinyemi O, Amirthalingam G, et al. Risk factors for SARS-CoV-2 among patients in the Oxford Royal College of General Practitioners Research and Surveillance Centre primary care network: a crosssectional study. Lancet Infect Dis. 2020. https://doi.org/10.1016/ S1473-3099(20)30371-6.

46. VanderWeele TJ, Hernán MA. Results on differential and dependent measurement error of the exposure and the outcome using signed directed acyclic graphs. Am J Epidemiol. 2012;175:1303-10.

47. Hernán MA, Cole SR. Invited Commentary: Causal diagrams and measurement bias. Am J Epidemiol 2009; 959-62; discussion 963-4

48. Sutton D, Fuchs K, D'Alton M, Goffman D. Universal screening for SARS-CoV-2 in women admitted for delivery. N Engl J Med. 2020. https://doi.org/10.1056/NEJMc2009316.

49. Ludvigsson JF. Systematic review of COVID-19 in children shows milder cases and a better prognosis than adults. Acta Paediatr. 2020;109:1088-95.

50. Cruz AT, Zeichner SL. COVID-19 in children: initial characterization of the pediatric disease. Pediatrics. 2020. https://doi. org/10.1542/peds.2020-0834.

51. Eyre DW, Lumley SF, O’Donnell D, Campbell M, Sims E, Lawson E, et al. Differential occupational risks to healthcare workers from SARS-CoV-2 observed during a prospective observational study. Elife. 2020. https://doi.org/10.7554/eLife.60675.

52. Krammer F, Simon V. Serology assays to manage COVID-19. Science. 2020;368:1060-1.

53. Volz E, Hill V, McCrone JT, Price A, Jorgensen D, O'Toole Á, et al. Evaluating the effects of SARS-CoV-2 spike mutation D614G on transmissibility and pathogenicity. Cell. 2021;184:6475.e11.

54. Leung K, Shum MH, Leung GM, Lam TT, Wu JT. Early transmissibility assessment of the N501Y mutant strains of SARS-CoV-2 in the United Kingdom, October to November 2020. Euro Surveill. 2021. https://doi.org/10.2807/1560-7917.ES.2020.26.1.2002106.

55. Hope Simpson RE. Infectiousness of communicable diseases in the household (measles, chickenpox, and mumps). Lancet. 1952;260:549-54.

56. Liu Y, Eggo RM, Kucharski AJ. Secondary attack rate and superspreading events for SARS-CoV-2. Lancet. 2020;395:e47.

57. Du Z, Xu X, Wu Y, Wang L, Cowling BJ, Meyers LA. Serial interval of COVID-19 among publicly reported confirmed cases. Emerg Infect Dis. 2020;26:1341-3.

58. Park YJ, Choe YJ, Park O, Park SY, Kim Y-M, Kim J, et al. Contact Tracing during coronavirus disease outbreak, South Korea, 2020. Emerg Infect Dis. 2020. https://doi.org/10.3201/eid26 10.201315 .

59. Zhu Y, Bloxham CJ, Hulme KD, Sinclair JE, Tong ZWM, Steele LE, et al. Children are unlikely to have been the primary source of household SARS-CoV-2 infections. Lancet Infect Dis. 2020. https://papers.ssrn.com/abstract $=3564428$

60. Tao J, Zhang X, Zhang X, Zhao S, Yang L, He D, et al. The time serial distribution and influencing factors of asymptomatic COVID-19 cases in Hong Kong. One Health. 2020;10:100166.

61. Jang S, Han SH, Rhee J-Y. Cluster of coronavirus disease associated with fitness dance classes, South Korea. Emerg Infect Dis J. 2020. https://wwwnc.cdc.gov/eid/article/26/8/20-0633_article

62. Voeten HA, Sikkema RS, Damen M, Oude Munnink BB, Arends $\mathrm{C}$, Stobberingh E, et al. Unravelling the modes of transmission of SARS-CoV-2 during a nursing home outbreak: looking beyond 
the church super-spread event. 2020. https://www.researchsquare. com/article/rs-63027/v1.pdf

63. Kim J, Choe YJ, Lee J, Park YJ, Park O, Han MS, et al. Role of children in household transmission of COVID-19. Arch Dis Child. 2020. https://doi.org/10.1136/archdischild-2020-319910.

64. Campbell F, Didelot X, Fitzjohn R, Ferguson N, Cori A, Jombart T. outbreaker2: a modular platform for outbreak reconstruction. BMC Bioinform. 2018;19:363.

65. Worby CJ, Lipsitch M, Hanage WP. Shared genomic variants: Identification Of Transmission Routes Using Pathogen DeepSequence data. Am J Epidemiol. 2017;186:1209-16.

66. Becker N. A general chain binomial model for infectious diseases. Biometrics. 1981;37:251-8.

67. Hong L-X, Lin A, He Z-B, Zhao H-H, Zhang J-G, Zhang C, et al. Mask wearing in pre-symptomatic patients prevents SARS-CoV-2 transmission: an epidemiological analysis. Travel Med Infect Dis. 2020;101803.

68. Zhang JZ, Zhou P, Han DB, Wang WC, Cui C, Zhou R, et al. Investigation on a cluster epidemic of COVID-19 in a supermarket in Liaocheng, Shandong province. Zhonghua Liu Xing Bing Xue Za Zhi. 2020;41:E055.

69. Kwok KO, Wong VWY, Wei WI, Wong SYS, Tang JW-T. Epidemiological characteristics of the first 53 laboratory-confirmed cases of COVID-19 epidemic in Hong Kong, 13 February 2020. Euro Surveill. 2020. https://doi.org/10.2807/1560-7917. ES.2020.25.16.2000155.

70. Burke RM, Midgley CM, Dratch A, Fenstersheib M, Haupt $\mathrm{T}$, Holshue M, et al. Active monitoring of persons exposed to patients with confirmed COVID-19-United States, January-February 2020. MMWR Morb Mortal Wkly Rep. 2020;69:245-6.

71. Zhang Z, Bi Q, Fang S, Wei L, Wang X, He J, et al. Insights into the practical effectiveness of RT-PCR testing for SARS-CoV-2 from serologic data, a cohort study. Epidemiology. medRxiv. 2020. https://www.medrxiv.org/content/10.1101/2020.09.01.20182 469v2.abstract

72. Waterfield T, Watson C, Moore R, Ferris K, Tonry C, Watt AP, et al. Seroprevalence of SARS-CoV-2 antibodies in children-a prospective multicentre cohort study. Pediatrics. medRxiv. 2020. https://www.medrxiv.org/content/10.1101/2020.08.31.20183 095v1.abstract

73. Qiu X, Nergiz AI, Maraolo AE, Bogoch II, Low N, Cevik M. Defining the role of asymptomatic SARS-CoV-2 transmission: a living systematic review. Epidemiology. medRxiv. 2020. https:// www.medrxiv.org/content/10.1101/2020.09.01.20135194v1.abstr act
74. Li W, Zhang B, Lu J, Liu S, Chang Z, Cao P, et al. The characteristics of household transmission of COVID-19. Clin Infect Dis. 2020. https://doi.org/10.1093/cid/ciaa450.

75. Wu J, Huang Y, Tu C, Bi C, Chen Z, Luo L, et al. Household Transmission of SARS-CoV-2, Zhuhai, China, 2020. Clin Infect Dis. 2020. https://doi.org/10.1093/cid/ciaa557.

76. Hu S, Wang W, Wang Y, Litvinova M, Luo K, Ren L, et al. Infectivity, susceptibility, and risk factors associated with SARS-CoV-2 transmission under intensive contact tracing in Hunan, China [Internet]. Infect Dis. (except HIV/AIDS). medRxiv. 2020. https ://doi.org/10.1101/2020.07.23.20160317

77. Epstein JB, Chow K, Mathias R. Dental procedure aerosols and COVID-19. Lancet Infect Dis. 2020. https://doi.org/10.1016/ S1473-3099(20)30636-8.

78. Cevik M, Tate M, Lloyd O, Maraolo AE, Schafers J, Ho A. SARS-CoV-2, SARS-CoV-1 and MERS-CoV viral load dynamics, duration of viral shedding and infectiousness: a living systematic review and meta-analysis. Infect Dis. (except HIV/AIDS). medRxiv. 2020. https://www.medrxiv.org/conte $\mathrm{nt} / 10.1101 / 2020.07 .25 .20162107 \mathrm{v} 2$.abstract

79. Xu Y, Li X, Zhu B, Liang H, Fang C, Gong Y, et al. Characteristics of pediatric SARS-CoV-2 infection and potential evidence for persistent fecal viral shedding. Nat Med. 2020;26:502-5.

80. Hua C-Z, Miao Z-P, Zheng J-S, Huang Q, Sun Q-F, Lu H-P, et al. Epidemiological features and viral shedding in children with SARS-CoV-2 infection. J Med Virol. 2020. https://doi. org/10.1002/jmv.26180.

81. Kissler SM, Fauver JR, Mack C, Tai C, Shiue KY, Kalinich CC, et al. Viral dynamics of SARS-CoV-2 infection and the predictive value of repeat testing. Epidemiology. medRxiv. 2020. https:// www.medrxiv.org/content/10.1101/2020.10.21.20217042v1.abstr act

82. Bi Q, Wu Y, Mei S, Ye C, Zou X, Zhang Z, et al. Epidemiology and transmission of COVID-19 in 391 cases and 1286 of their close contacts in Shenzhen, China: a retrospective cohort study. Lancet Infect Dis. 2020. https://doi.org/10.1016/S1473 $-3099(20) 30287-5$.

83. Hu M, Lin H, Wang J, Xu C, Tatem AJ, Meng B, et al. The risk of COVID-19 transmission in train passengers: an epidemiological and modelling study. Clin Infect Dis. 2020. https://doi. org/10.1093/cid/ciaa1057.

Publisher's Note Springer Nature remains neutral with regard to jurisdictional claims in published maps and institutional affiliations. 